\title{
Uso da suavização da fronteira na determinação de pesos únicos em modelos DEA CCR
}

\author{
Eliane Ribeiro Pereira ${ }^{a}$, João Carlos Correia Baptista Soares de Mello ${ }^{\text {b* }}$ \\ aUniversidade Federal do Rio de Janeiro, Rio de Janeiro, RJ, Brasil \\ b*Universidade Federal Fluminense, Niterói, RJ, Brasil, jcsmello@producao.uff.br
}

\begin{abstract}
Resumo
Ao longo do tempo, o número e o campo de aplicações em DEA aumentaram sobremaneira. A complexidade decorrente das relações entre os múltiplos inputs e outputs das Unidades Tomadoras de Decisão (DMUs) em estudo dificulta o uso de outras metodologias, tornando a ferramenta DEA bastante atrativa na busca da identificação da eficiência dessas DMUs. 0 objetivo deste estudo é definir um método de resolução da ambiguidade na determinação dos pesos das DMUs extremo-eficientes, de forma que cada DMU tenha um conjunto único de pesos, permitindo conhecer a importância relativa de cada input ou output. A proposta é substituir a fronteira DEA CCR original, que é linear por partes, por uma fronteira suavizada, o mais próximo possível da primeira, mas continuamente diferenciável em todos os seus pontos. A suavização possibilita a realização de aplicações como o cálculo dos trade-offs nas DMUs extremo-eficientes e a Avaliação Cruzada.
\end{abstract}

Palavras-chave

DEA. Fronteira suavizada. Avaliação cruzada.

\section{Introdução}

A Análise de Envoltória de Dados (Data Envelopment Analysis - DEA) é um método de apoio à decisão desenvolvido por Charnes et al. (1978), com o objetivo de comparar unidades produtivas (Decision Making Units - DMUs) que realizam tarefas similares, utilizando quantidades de recursos disponíveis (inputs) e produzindo diferentes saídas (outputs). A metodologia DEA permite identificar as DMUs eficientes, medir e localizar a ineficiência, além de estimar uma função de produção linear por partes (piece-wise linear frontier), que fornece o benchmark (referência) para as DMUs ineficientes.

A Análise de Envoltória de Dados (Data Envelopment Analysis - DEA) facilita a modelagem da complexidade do mundo real, que suscita enfoques e interesses diversificados. Assim, é uma ferramenta matemática fundamental na realização de estudos voltados a investigar a eficiência de unidades produtivas.

0 método constrói uma fronteira de eficiência, cujos vértices são formados pelas DMUs Pareto eficientes, aquelas que possuem a melhor relação produto/insumo. As menos eficientes ficam situadas numa região inferior à fronteira. Nesses vértices, por não existir derivada, ocorre uma descontinuidade, o que impossibilita o cálculo de um valor único para os multiplicadores dessas DMUs.

Tal fato é um problema na aplicação prática da metodologia DEA. Sendo um conceito relativo, a eficiência DEA é calculada através da comparação das práticas de uma DMU com relação à das demais, que realizam tarefas semelhantes. Justamente das DMUs que formam os vértices da fronteira de eficiência, consideradas detentoras das melhores práticas de gestão, não é possível compreender a forma como atuam, em particular as razões de substituição entre inputs e outptus.

0 assunto é tratado de diferentes formas na literatura. Diversos autores trataram do problema de múltiplas soluções nas DMUs extremo-eficientes em outros contextos, sem que fossem mantidas as mesmas propriedades da fronteira DEA. Soares de 
Mello (2002) e depois Nacif et al. (2009) propuseram a suavização da fronteira de eficiência, para os modelos DEA BCC N-dimensionais, que pressupõem a condição de convexidade na fronteira. Para os modelos CCR, foi proposta apenas uma solução parcial, já que a condição de proporcionalidade entre inputs e outputs dificulta demasiadamente o problema.

Este artigo apresenta uma metodologia para a determinação de um valor único para os multiplicadores do Modelo DEA CCR, baseada nos trabalhos desenvolvidos por Soares de Mello (2002) e Nacif et al. (2009). Propõe-se, tal como nas referências anteriores, o estabelecimento de uma nova fronteira de eficiência, que mantém as propriedades da fronteira original, mas na qual é possível calcular derivadas parciais em todos os seus pontos. Para manter as propriedades do modelo CCR, é necessária a utilização de uma função aproximante homogênea. A proposta é utilizar como função aproximante um polinômio homogêneo único, de grau suficientemente elevado, de forma a garantir a viabilidade do modelo proposto.

0 artigo foi organizado da seguinte forma: a seção 2 apresenta uma breve discussão acerca do problema de multiplicidade de pesos para o Modelo dos Multiplicadores. Na seção 3, são apresentadas algumas características e propriedades do modelo CCR, fundamentais para a construção do modelo de suavização DEA CCR proposto na seção seguinte. $\mathrm{Na}$ seção 4, é desenvolvido o modelo de suavização para a fronteira DEA CCR. Nas seções 5 e 6, apresentam-se o cálculo dos pesos em fronteiras suavizadas e seu uso em avaliação cruzada. Em seguida, é apresentado um exemplo numérico seguido das principais conclusões do trabalho.

\section{Conjunto de pesos do Modelo dos Multiplicadores}

A metodologia DEA permite estimar a eficiência técnica de unidades produtivas sem recorrer ao arbítrio de pesos para cada variável de input ou output, e sem que seja necessário converter todas as variáveis em valores econômicos comparáveis (Lins \& Angulo-Meza, 2000).

Os modelos DEA clássicos apresentam duas formulações equivalentes (Cooper et al., 2000). A primeira, conhecida como modelo do Envelope, define uma região viável de produção e trabalha com uma distância (não euclidiana) de cada DMU à fronteira desta região. Ela fornece os coeficientes de ponderação de cada DMU eficiente na formação da DMU virtual, que serve de benchmark para cada
DMU ineficiente. A segunda, conhecida como Modelo dos Multiplicadores, trabalha com a razão de somas ponderadas de produtos e recursos, fornecendo os coeficientes de ponderação que cada DMU atribui a cada input e output. As duas formulações constituem problemas duais, fornecendo, portanto, a mesma eficiência para cada DMU (Soares de Mello et al., 2004).

A ferramenta DEA concede liberdade total para que cada unidade de tomada de decisão fixe os pesos (multiplicadores) que julgar apropriado a cada input e output, desde que esses pesos, quando aplicados às demais DMUs, não gerem uma razão superior à unidade. Assim, são valorizados os aspectos mais favoráveis a cada DMU, o que torna o modelo excessivamente flexível. Segundo Soares de Mello et al. (2006), tal prática minimiza a possibilidade de tendenciosidade e tende a eliminar a subjetividade da análise. Soares de Mello et al. (2002) mencionam o desenvolvimento de técnicas que permitem aumentar o poder de discriminação do método e incorporar a análise dos pesos ou multiplicadores, como a Avaliação Cruzada e Restrições aos pesos.

A fronteira de eficiência gerada pela metodologia DEA é linear por partes, e, em seus vértices, ocorre uma descontinuidade, já que neles não existe derivada. Assim, a solução do Modelo dos Multiplicadores conduz a múltiplas soluções ótimas para as DMUs extremo-eficientes. 0 teorema da folga complementar permite mostrar que os multiplicadores gerados na solução do modelo são os coeficientes da equação do hiperplano tangente à fronteira no ponto de projeção da DMU em estudo (Lins \& Angulo-Meza, 2000). Nas DMUs extremo-eficientes, por não existirem derivadas, não existe hiperplano tangente, embora exista uma infinidade de hiperplanos suporte. Tem-se, portanto, uma infinidade de multiplicadores para cada DMU extremo-eficiente, todos eles conduzindo à eficiência 1 para essas DMUs. Tal fato impossibilita o cálculo de um valor único para os multiplicadores dessas DMUs, impedindo o conhecimento das razões de substituição (tradeoffs) entre os diversos inputs, ou o quanto a variação de um input afeta a variação de um determinado output. Além disso, a ausência de valores únicos para os pesos das DMUs extremoeficientes é um obstáculo ao uso de DEA como ferramenta auxiliar em problemas multicritério, quando se deseja atribuir pesos aos critérios sem julgamentos de valor do decisor.

Nacif et al. (2009) destacam diferentes abordagens realizadas por pesquisadores para o problema da não unicidade dos multiplicadores para as DMUs extremo-eficientes, cujas soluções se mostraram 
deficientes. Devido à natureza linear por partes da fronteira DEA, Rosen et al. (1998) consideraram ser impossivel contornar o problema da multiplicidade de valores e propuseram a adoção de um quadro simplex modificado para calcular os limites de variação dos multiplicadores, que, segundo a proposta, poderiam variar entre o valor calculado com base na derivada à esquerda e o calculado com base na derivada à direita.

Charnes et al. (1985) propuseram o uso de um valor único arbitrário para as derivadas, a partir do cálculo de uma média ponderada, baseado nos baricentros das hipersuperfícies concorrentes. Tal proposta esbarra na necessidade de conhecer as equações de todas as faces, que é um problema computacionalmente intenso. Além disso, existe a possibilidade de ocorrerem variações bruscas devido à descontinuidade das derivadas; e a impossibilidade da solução proposta ser aplicada a DMUs que marquem o início da região Pareto ineficiente, ou que sejam adjacentes a uma face de dimensão incompleta.

Andersen \& Petersen (1993) propuseram um modelo que não limita as eficiências ao intervalo $[0,1]$ e que elimina uma restrição diferente no PPL de cada DMU. Isso significa dizer que a fronteira eficiente apresenta uma configuração diferente, dependendo da DMU analisada (Soares de Mello et al., 2004).

Cooper et al. (2007) propuseram um procedimento de duas etapas para resolver o problema de degeneração do modelo do envelope. A ideia do procedimento é explorar o conjunto de alternativas ótimas, a fim de ajudar a efetuar a escolha de pesos ótimos. Na primeira etapa, eles analisam os pesos associados com hiperplanos suportados pelo número máximo de unidades extremo-eficientes. Na segunda etapa, são escolhidos os pesos que maximizam o valor relativo de inputs e outputs selecionados para a avaliação de eficiência, com a garantia de que nenhuma variável seja completamente ignorada na avaliação de eficiência das unidades.

Soares de Mello (2002) e Soares de Mello et al. (2002, 2004) mostraram ser possível contornar a impossibilidade mencionada por Rosen et al. (1998), mantendo-se as propriedades da fronteira de eficiência. Propuseram a substituição da fronteira DEA original, por uma fronteira suavizada, continuamente diferenciável, respeitando as propriedades básicas de DEA para os modelos CCR e BCC, com até três dimensões. Para o modelo BCC, a técnica foi proposta em termos gerais, desde que o número de inputs ou de outputs fosse unitário. Já para o modelo CCR a técnica proposta, embora tecnicamente correta, só conseguia ser aplicada em casos muito particulares, devido à complexidade dos cálculos exigidos, mesmo em situações de apenas 3 dimensões.
Nacif et al. (2009) usaram o mesmo referencial teórico para expandir os resultados obtidos por Soares de Mello (2002) e Soares de Mello et al. (2002, 2004), estudando a suavização da fronteira DEA para o modelo BCC N-dimensional, com múltiplos inputs e outputs, garantindo, assim, maior aplicabilidade à técnica. 0 modelo proposto por Nacif et al. (2009) permite determinar valores únicos para os multiplicadores usados por cada DMU eficiente, preservando a fronteira tão próxima quanto possível da original, além de manter a liberdade de cada DMU determinar os seus próprios multiplicadores. Esta generalização abriu caminho para o uso de outras vantagens da suavização, como a substituição de todas as faces por uma única face. Uma aplicação desta técnica pode ser vista em Gomes et al. (2004), na qual a suavização é usada para viabilizar alguns cálculos na aplicação do modelo de ganhos de soma zero, de lins et al. (2003). Outra aplicação pode ser vista em Brandão et al. (2013), que introduz um relaxamento das restrições para verificar quais das DMUs indicadas como eficientes pelo modelo BCC o são realmente. Alguns problemas de convexidade e viabilidade das soluções, presentes nas formulações originais da suavização, foram resolvidos por Brandão \& Soares de Mello (2013) e Brandão (2013).

Os modelos de suavização aqui descritos não são a única fronteira baseada em DEA que apresenta derivadas em todos os pontos. Embora em um contexto totalmente diferente (o da distribuição de recursos e/ou produtos), os chamados modelos DEA paramétricos também apresentam uma fronteira eficiente continuamente diferenciável. Estes modelos podem ser vistos em Milioni \& Alves (2013), Guedes et al. (2012), Silva \& Milioni (2012), Milioni et al. (2011a, b), Avellar et al. (2010), Colen et al. (2009), Avellar et al. (2007), e Silveira et al. (2011).

\section{Modelo CCR}

0 modelo CCR, também conhecido como CRS - Constant Returns to Scale, foi desenvolvido por Charnes et al. (1978) e constrói uma superfície linear por partes, não paramétrica, envolvendo os dados. A forma como é feita a projeção determina a orientação do modelo DEA que pode ter orientação a inputs e a outputs. 0 modelo é dito com orientação a inputs, quando a eficiência é atingida por uma redução equiproporcional de entradas, mantidas as saídas constantes; e com orientação a outputs, quando se deseja maximizar os resultados, sem diminuir os recursos (Angulo-Meza, 1998).

0 Modelo CCR (1), desenvolvido inicialmente com orientação a inputs, trabalha com retornos 
constantes de escala, isto é, qualquer variação nos insumos (inputs) resulta em uma variação proporcional nos produtos (outputs). Sua definição original considera a maximização da eficiência $h_{\mathrm{o}}$ da unidade produtiva. É chamado Modelo dos Multiplicadores, por determinar os coeficientes ("pesos") dos produtos e dos recursos.

$\operatorname{Max} h_{\mathrm{o}}=\sum_{r=1}^{s} u_{\mathrm{r}} y_{\mathrm{ro}}$

\section{Sujeito a}

$\sum_{i=1}^{m} v_{\mathrm{i}} X_{\mathrm{io}}=1$

$\sum_{r=1}^{s} u_{\mathrm{r}} y_{\mathrm{rj}}-\sum_{i=1}^{m} v_{\mathrm{i}} x_{\mathrm{ij}} \leq 0, j=1, \ldots, n$

$u_{\mathrm{r}}, v_{\mathrm{i}} \geq 0, \forall r, i$

onde $v_{\mathrm{i}}$ e $u_{\mathrm{r}}$ são os multiplicadores dos inputs $i, i=1, \ldots$, $m$, e outputs $r, r=1, \ldots$, s, respectivamente; $x_{\mathrm{ij}}$ e $y_{\mathrm{rj}}$ são os inputs $i$ e outputs $r$ da DMU $j, j=1, \ldots, n ; x_{\text {io }} \mathrm{e}$ $y_{\text {ro }}$ são os inputs $i$ e outputs $r$ da DMU 0. Uma DMU é eficiente quando $h_{0}=1$, o que significa dizer que a restrição relativa a essa DMU está ativa e, portanto, tem folga igual a zero.

Esse modelo é uma generalização do trabalho de Farrell (1957) para múltiplos insumos e múltiplos produtos, no qual se determina a eficiência através da divisão entre a soma ponderada dos produtos (output virtual) pela soma ponderada dos insumos (input virtual), construindo uma superfície linear por partes, não paramétrica, sobre os dados. No lugar de uma ponderação igual para todas as DMUs, o modelo permite a escolha de pesos para cada variável, da forma que lhe seja mais favorável, desde que esses pesos, quando aplicados às demais DMUs, não gerem uma razão superior à unidade.

Para as DMUs eficientes, diferentes conjuntos de pesos para seus inputs fornecem a mesma solução para o PPL que calcula a eficiência da DMU em estudo. A teoria de Programação Linear mostra que se um PPL tem mais de uma solução ótima, então tem uma infinidade. Assim, existe uma infinidade de conjuntos de pesos ótimos que tornam a DMU em estudo eficiente. Como já visto isso acontecerá para todas as DMUs extremo-eficientes (vértices da fronteira).

Com base no Modelo dos Multiplicadores (primal) é possível desenvolver o seu dual, conhecido como Modelo do Envelope, que, pelo teorema da dualidade forte, apresentará o mesmo valor ótimo para a função objetivo, quando esse existir (Bregalda \& Bornstein, 1981).
Pode-se, ainda, desenvolver um modelo orientado a outputs, ou seja, que maximiza as saídas, mantendo inalteradas as entradas. Neste modelo, as variáveis de decisão são as mesmas do modelo (1), orientado a inputs, sendo que $h$ representa por quanto todos os produtos devem ser multiplicados, mantendo-se constantes os recursos, para a DMU $o$ atingir a fronteira eficiente. Com a orientação a output, $h_{0}$ sempre assume valores superiores à unidade, por isso, a eficiência passa a ser definida como sendo o inverso de $h_{0}$.

Os modelos CCR orientados a input e a output fornecem o mesmo valor de eficiência, identificam o mesmo conjunto de DMUs eficientes e ineficientes, estimando, assim, a mesma fronteira eficiente.

0 modelo CCR trabalha com retorno constante de escala, isto é, se existir uma DMU $(x, y)$ com atividade viável de produção, então, existirá outra DMU $(\lambda x, \lambda y)$, cuja operação também é viável. Além disso, sua propriedade de proporcionalidade impõe que quaisquer variações nos inputs, impliquem variações proporcionais nos outputs. 0 modelo de suavização proposto neste estudo pressupõe o uso de uma função aproximante para substituir a fronteira original do problema, que seja tão próxima quanto possível dessa fronteira original e que mantenha suas propriedades originais. As propriedades inerentes aos modelos CCR dificultam sobremaneira a escolha de funções aproximantes, já que a necessidade de garantir a proporcionalidade entre inputs e outputs requer a adoção de funções homogêneas de $1^{\circ} \mathrm{grau}$, o que reduz muito a gama de possibilidades. A solução proposta para esse problema está apresentada na seção 4.

\section{Suavização da fronteira DEA CCR}

Como mencionado anteriormente, o Modelo dos Multiplicadores conduz a múltiplas soluções ótimas para as DMUs extremo-eficientes, impossibilitando o cálculo das taxas de substituição (tradeoffs) entre diversos inputs, ou do quanto a variação de um input afeta a variação de um determinado output, o que é uma informação fundamental em problemas de eficiência. Outras informações relevantes, como a importância de cada variável ou a determinação de preços sombra, ficam igualmente comprometidas. 0 que se procura é um método de resolução desta ambiguidade na determinação dos pesos das DMUs extremo-eficientes, de forma que cada DMU tenha um conjunto único de pesos, permitindo conhecer a importância relativa de cada input ou output.

0 desenvolvimento da técnica considera uma pseudométrica que trata da proximidade simultânea 
das funções e suas derivadas, uma vez que a fronteira suavizada deve estar não só próxima da original, como também possuir derivadas semelhantes às dela, onde existirem as derivadas (Soares de Mello, 2002; Soares de Mello et al., 2002, 2004). No caso bidimensional, deve-se considerar o fato de que a região da fronteira que contém 2 DMUs eficientes consecutivas é um segmento de reta - que é o menor comprimento de arco entre dois pontos. Além disso, derivadas de valor muito diferente da inclinação da reta suporte do segmento implicam maior comprimento de arco. Dessa forma, a minimização do comprimento de arco da fronteira suavizada é suficiente para garantir a sua proximidade com a fronteira original, uma vez que esta última é composta apenas por segmentos de reta.

0 comprimento de arco é representado por:

$\int_{x_{1}}^{x_{2}} \sqrt{1+\left(\frac{d f}{d x}\right)^{2} d x}$

onde $y=f(x)$ é a equação da fronteira suavizada.

Os mesmos argumentos podem ser generalizados para problemas de dimensão superior, substituindo-se segmento de reta por uma região do hiperplano e integral simples por integral múltipla.

\subsection{Formulação original do Modelo de Suavização}

$\mathrm{Na}$ formulação proposta por Soares de Mello et al. (2002), foram considerados modelos DEA com apenas 1 output (o mesmo podendo ser realizado em modelos com 1 input), e a suavização consiste em procurar uma função que minimize o comprimento de arco (ou sua generalização $n$-dimensional), que contenha as DMUs Pareto eficientes e que tenha derivadas parciais de segunda ordem em todos os pontos. Por facilidade computacional, minimizou-se o quadrado do comprimento de arco, sem que o resultado fosse alterado. Então, após determinar as DMUs extremo-eficientes no modelo DEA clássico, a suavização foi obtida pelo problema variacional (3).

$$
\begin{aligned}
& \min L=\int_{R}\left[1+\sum_{i}\left(\frac{\partial F}{\partial x_{i}}\right)^{2}\right] d S \\
& \text { Sa } \\
& F\left(\vec{X}_{j}\right)=\text { output }\left(\vec{X}_{j}\right), \forall \vec{X}_{j} \in E=\{\vec{X}: \vec{X} \text { é DMU Pareto eficiente }\} \\
& \left.\forall \vec{X}_{j} \exists \frac{\partial F}{\partial x_{i}}\right|_{\vec{X}}=\vec{X}_{j}
\end{aligned}
$$

Soares de Mello et al. (2004) destacam a importância de que na última restrição de (3) seja garantida, em especial, a existência das derivadas nos pontos correspondentes às DMUs extremo-eficientes, por serem estes os pontos de descontinuidade na fronteira original. São necessárias condições de contorno adicionais a serem obtidas das propriedades de cada modelo DEA.

Para o modelo CCR, a condição de convexidade do modelo BCC é substituída pela de proporcionalidade: aumentos (diminuições) proporcionais nos inputs provocam um aumento (diminuição) proporcional no output. Ou seja, $F(k \cdot \vec{X})=k \cdot F(\vec{X})$, o que significa que $F$ tem que ser homogênea de grau 1 (COELl et al., 1998). Assim sendo, deve respeitar o teorema de Euler para funções homogêneas:

$\vec{X} \cdot \vec{\nabla} F=n F$

como $\mathrm{n}=1, \vec{X} \cdot \vec{\nabla} F=F$

Esta é a restrição adicional que $F$ deve satisfazer no modelo CCR (Soares de Mello, 2002). Assim, se houver $n$ DMUs Pareto-eficientes, haverá $n$-1 funções aproximantes, $2 n-2$ restrições de passagem pelas DMUs e $n-2$ restrições de suavidade, havendo, portanto, mais variáveis de decisão que restrições.

Assim sendo, na suavização de fronteiras DEA, a função diferenciável que substitui a fronteira CCR deve ser homogênea de grau 1, o que restringe muito a escolha de possíveis aproximantes. Por outro lado, tal imposição permite aproveitar as propriedades das funções homogêneas para simplificar o problema.

Para o modelo CCR tridimensional - dois inputs e um output, ou um input e dois outputs, a fronteira pode ser reduzida a duas dimensões cortando-se a superfície por um plano de nível de cota 1. Como a fronteira CCR é determinada por funções homogêneas de $1^{\circ}$ grau, algebricamente, esse procedimento equivale a dividir tanto os inputs quanto o output pelo valor do output (ou o input e os outputs pelo valor do input), como mostrado por Soares de Mello (2002).

0 uso da minimização do comprimento de arco conduz, neste caso, ao cálculo de integrais duplas, cujos limites de integração dependem do seu resultado, o que torna sua aplicação bastante difícil. Soares de Mello (2002) propôs um problema MinMax, no qual primeiramente realizava-se a minimização da maior das diferenças entre a fronteira original e a suavizada nas DMUs eficientes e, a seguir, no ponto médio de cada segmento da fronteira original. 0 modelo, que requer pelo menos três DMUs eficientes para poder ser aplicado, possuía, ainda, uma restrição extra que garantia que a fronteira suavizada passasse abaixo dos pontos da fronteira original. 


\subsection{Formulação do Modelo de Suavização DEA CCR}

0 modelo de suavização proposto por Soares de Mello (2002) foi exemplificado para o caso tridimensional (2 inputs e 1 output ou 1 input e 2 outputs), devido à dificuldade envolvida nos cálculos. Dessa forma, decidiu-se investigar melhor a suavização para modelos DEA CCR, com base na metodologia proposta por Nacif et al. (2009).

Para se resolver o problema, é necessário interpretar o que significam os pesos, o que é melhor compreendido no Modelo do Envelope, problema dual do Modelo dos Multiplicadores. Pelo teorema das folgas complementares, é possível mostrar que os pesos correspondem aos coeficientes do hiperplano, que é tangente a cada ponto da fronteira eficiente - denominado hiperplano suporte (Lins \& Angulo-Meza, 2000).

No modelo CCR, a equação do hiperplano tangente à projeção radial da DMU k é dada por:

$\sum_{j} u_{j k}^{*} \cdot y_{j}-\sum_{i} v_{i k}^{*} \cdot x_{i}=0$

onde $x_{\mathrm{ik}}$ são os inputs, $y_{\mathrm{jk}}$ são os outputs, $v_{\mathrm{ik}}^{*}$ e $u_{\mathrm{jk}}^{*}$ são os coeficientes de inputs e outputs que maximizam a eficiência. Assim, pode-se perceber que o problema de se determinar um conjunto único de pesos no modelo dos multiplicadores equivale a determinar um hiperplano tangente no modelo do envelope (Soares de Mello et al., 2002), ou seja, ser possível calcular as derivadas em todos os pontos da fronteira. Mas essa fronteira tem derivadas descontínuas, já que é linear por partes, impossibilitando o cálculo de um valor único para os multiplicadores de algumas DMUs.

Este estudo propõe resolver o problema através da substituição da fronteira calculada no modelo DEA, por outra que admita hiperplano tangente em todos os seus pontos, mas que mantenha a maior parte das propriedades da fronteira DEA original, tal como possuir as mesmas DMUs eficientes. Como os únicos pontos da fronteira em que não existe derivada são as DMUs que representam vértices da hipersuperfície, o que se procura é suavizar esses vértices. Embora os vértices sejam bem identificados em representação geométrica, a sua determinação algébrica faz uso do modelo de supereficiência (Andersen \& Petersen, 1993). Os vértices correspondem às DMUs que no modelo de supereficiência obtêm valor superior à unidade ou inviabilizam o PPL e são denominados DMUs extremo-eficientes (Charnes et al., 1978).

A proposta é suavizar a fronteira do modelo DEA CCR representando-a por uma equação que relacione todos os inputs e outputs. Propõe-se o uso de um polinômio homogêneo único como aproximante da fronteira DEA CCR, de forma a preservar a propriedade de homogeneidade requerida para este modelo. Define-se, então, a função U que relaciona os polinômios homogêneos de outputs e de inputs, da seguinte forma:

$$
\begin{aligned}
& U\left(x_{1}, x_{2}, \ldots, x_{n}, z_{1}, z_{2}, \ldots, z_{n}\right)= \\
& F_{g_{l, n}}\left(x_{1}, x_{2}, \ldots, x_{n}\right)-H_{g_{o, m}}\left(z_{1}, z_{2}, \ldots, z_{m}\right)
\end{aligned}
$$

onde, F e H representam os polinômios homogêneos de inputs e outputs, respectivamente; $g_{1}$ é o grau do polinômio dos inputs e $\mathrm{g}_{0}$, o grau do polinômio dos outputs; $n$ representa o número de variáveis de entrada (número de inputs) e $m$ o número de variáveis de saída (número de outputs). Finalmente, $x_{\mathrm{i}}$ e $z_{\mathrm{i}}$ são os valores dos inputs e outputs do problema em estudo.

Deve-se ressaltar que outras expressões funcionais, em particular as cônicas e suas generalizações, poderiam ser melhor adaptadas às exigências da homogeneidade. No entanto, a pequena quantidade de parâmetros nas suas equações poderia inviabilizar que todas as restrições de igualdade fossem satisfeitas. Embora haja técnicas para superar esse problema (Soares de Mello et al., 2002), a complexidade dos cálculos aumentaria muito com o seu uso. Acresce que os cálculos das derivadas e integrais ficariam extremamente trabalhosos (e alguns casos mesmo inviáveis de se fazer literalmente) com essas funções. Assim, optou-se pelo uso de polinômios, que já mostraram bons resultados em problemas anteriores de suavização e em problemas de transmissão de calor baseados em equações semelhantes (Soares de Mello, 1987). Com efeito, os cálculos de derivadas e integrais tornam-se triviais com o uso de polinômios e, além disso, com a escolha adequada do seu grau garante-se a viabilidade de cumprir todas as restrições de igualdade. Por outro lado, é preciso cuidado em não extrapolar resultados já que as propriedades impostas aos polinômios apenas são válidas na região definida, podendo apresentar variações muito bruscas tão logo se saia da região em que as propriedades dos polinômios foram definidas.

Com as funções polinomiais definidas em (6), tem-se a representação de uma superfície de nível $U=F-H$, cujo análogo multidimensional do comprimento de arco deverá ser minimizado, como mostrado no modelo (3). 0 uso da função U permite que as restrições de suavização sejam satisfeitas implicitamente pelas derivadas da função $U$, que podem ser facilmente calculadas.

A formulação proposta considera a diferenciação de todas as entradas em relação a todas as saídas, 
assim como todas as saídas em relação a todas as entradas:



$$
\begin{aligned}
& S a \\
& U\left(x_{\text {lefi }} \ldots x_{\text {nef }}, z_{\text {lef }} \ldots z_{\text {mefi }}\right)=0, \quad \forall D M U \text { extremo-eficiente } \quad \text { (7.I) } \\
& \frac{\partial U}{\partial x_{i}}\left(x_{\text {max }}, \ldots, x_{n \max }\right) \geq 0, \forall i=1, \ldots, n \\
& \frac{\partial U}{\partial z_{i}}\left(z_{l \min }, \ldots, z_{n \min }\right) \leq 0, \forall i=1, \ldots, m \\
& \frac{\partial U}{\partial x_{i}} \geq 0, \forall D M U \text { extremo-eficiente } \\
& \frac{\partial U}{\partial z_{i}} \leq 0, \forall D M U \text { extremo-eficiente }
\end{aligned}
$$

A formulação (7) representa a proposta para o modelo geral DEA CCR suavizado para quaisquer números de variáveis de entrada e saída. A função objetivo é dada pela integral $(\mathrm{n}+\mathrm{m})$-upla, onde $x_{\text {imin }}$ e $x_{\text {imax }}$ representam o menor e o maior valor de cada input; e $z_{\text {imin }}$ e $z_{\text {imax }}$, o menor e o maior valor de cada output, respectivamente. A restrição (7.1) garante que as DMUs extremo-eficientes estejam contidas na fronteira suavizada. A restrição (7.2) garante a monotonicidade crescente da fronteira para os inputs em seus pontos máximos, enquanto para os outputs é garantida através de (7.3) em seus pontos mínimos. $\mathrm{Na}$ formulação do modelo de suavização para o caso BCC (Nacif et al., 2009), às restrições mencionadas são adicionadas restrições de convexidade da fronteira, propriedade a ser garantida para esse tipo de modelo, de forma a garantir a não ocorrência de grandes variações na função aproximante a ser definida. Em modelos CCR, a complexidade para garantir a não ocorrência de tais variações requer a inclusão das restrições (7.4) e (7.5), a fim de garantir a monotonicidade para inputs e outputs nas DMUs extremo-eficientes. Como o interesse do estudo é calcular os trade-offs nas DMUs extremo-eficientes, esse modelo garante tal possibilidade.

0 modelo em estudo foi desenvolvido para quaisquer números de inputse outputs e interpretado geometricamente para problemas com um output (ou um input). Sem perda de generalidade, a fim de facilitar a compreensão da proposta, o problema será apresentado a partir de um exemplo genérico que possua 2 inputs e 1 output, de grau $n$. Assim, tem-se:

$U=a_{1} x_{1}^{n}+a_{2} x_{1}^{n-1} x_{2}+a_{3} x_{1}^{n-2} x_{2}^{2}+a_{4} x_{1}^{n-3} x_{2}^{3}+\ldots+a_{n+1} x_{2}^{n}-z_{1}^{n}$

onde $x_{1}, x_{2}$ e $z_{1}$ são, respectivamente, os valores dos inputs e do output da DMU em análise; e $a_{\mathrm{i}}, i=1,2$, ..., $n+1$ são os coeficientes do polinômio homogêneo aproximante a ser determinado.

As derivadas parciais de primeira ordem de $\mathrm{U}$ serão dadas por: $\frac{\partial U}{\partial x_{1}}=n a_{1} \frac{d U}{d x_{1}}+(n-1) a_{2} \frac{d U}{d x_{1}}+(n-2) a_{3} \frac{d U}{d x_{1}}+(n-3) a_{4} \frac{d U}{d x_{1}}+\ldots+a_{n} \frac{d U}{d x_{1}}$

$\frac{\partial U}{\partial x_{2}}=a_{2} \frac{d U}{d x_{2}}+2 a_{3} \frac{d U}{d x_{2}}+3 a_{4} \frac{d U}{d x_{2}}+\ldots+n a_{n+1} \frac{d U}{d x_{2}}$

$\frac{\partial U}{\partial z_{1}}=-n \frac{d U}{d z_{1}}$

Como no caso do exemplo proposto $\frac{d U}{d z_{1}}$ será sempre não negativo, as restrições (7.3) e (7.5) poderão ser suprimidas, já que serão verdadeiras para quaisquer valores de $z_{i^{*}}$.

\section{Cálculo dos pesos}

A substituição da fronteira linear por partes por outra suavizada, derivável em todos os seus pontos, permite a realização do cálculo dos pesos ou multiplicadores únicos para todas as DMUs, que, pelo teorema das folgas complementares, podem ser interpretados como os coeficientes da equação do hiperplano tangente (Lins \& Angulo-Meza, 2000).

Considerando-se o modelo geral de suavização da fronteira CCR proposto em (7), onde a função $U$ relaciona os polinômios homogêneos de outputs $(\mathrm{H})$ e de inputs (F), a equação do hiperplano tangente à hipersuperfície de nivel $U\left(x_{1}, x_{2}, \ldots, x_{n}, z_{1}, z_{2}, \ldots, z_{n}\right)=0$, num ponto $\left(x_{1 \mathrm{k}}, \ldots, x_{\mathrm{nk}}, z_{1 \mathrm{k}}, \ldots, z_{\mathrm{mk}}\right)$ encontra-se relacionada em (10), devendo-se calcular todas as derivadas nesse ponto.

$\sum_{i=1}^{n}\left(\left(x_{i}-x_{i k}\right) \frac{\partial U}{\partial x_{i}}\right)+\sum_{j=1}^{m}\left(\left(z_{j}-z_{j k}\right) \frac{\partial U}{\partial z_{j}}\right)=0$

A expressão (10) pode ser escrita como em (11):

$$
\sum_{j=1}^{m}\left(z_{j} \frac{\partial U}{\partial z_{j}}\right)+\sum_{i=1}^{n}\left(x_{i} \frac{\partial U}{\partial x_{i}}\right)=\sum_{j=1}^{m}\left(z_{j k} \frac{\partial U}{\partial z_{j}}\right)+\sum_{i=1}^{n}\left(x_{i k} \frac{\partial U}{\partial x_{i}}\right)
$$

Considerando-se que no modelo CCR, a equação do hiperplano tangente à projeção radial da DMU $k$ é dada por (5), onde $x_{\mathrm{i}}$ e $z_{\mathrm{j}}$ podem representar as coordenadas da DMU $k$ se ela for eficiente, ou as coordenadas da DMU alvo, caso $k$ não seja eficiente. As equações de suavização permitem calcular a equação da reta tangente em cada ponto, cujo coeficiente angular permite a determinação de uma relação entre os coeficientes de inputs e outputs, $v_{\mathrm{ik}}$ e $u_{\mathrm{jk}}$.

Assim, a análise das equações (5) e (11), ambas representando o hiperplano tangente à fronteira suavizada, permite escrever (12) a (15): 
$u_{j k}=\frac{\partial U}{\partial z_{j}}$

$v_{i k}=-\frac{\partial U}{\partial x_{i}}$

$\sum_{j=1}^{m}\left(z_{j k} \frac{\partial U}{\partial z_{j}}\right)+\sum_{i=1}^{n}\left(x_{i k} \frac{\partial U}{\partial x_{i}}\right)=0$

ou,

$\sum_{i=1}^{n}\left(x_{i k} \frac{\partial U}{\partial x_{i}}\right)=-\sum_{j=1}^{m}\left(z_{j k} \frac{\partial U}{\partial z_{j}}\right)$

0 PPL do Modelo DEA CCR dos Multiplicadores com orientação a inputs apresentado em (1) possui a restrição de igualdade $\sum_{i=1}^{n} v_{i} x_{i k}=1$, que precisa ser respeitada. Para tanto, deve-se dividir cada equação por $\sum_{i=1}^{n}\left(x_{i k} \frac{\partial U}{\partial x_{i}}\right)$ :

$u_{j k}=\frac{\frac{\partial U}{\partial z_{j}}}{-\sum_{i=1}^{n}\left(x_{i k} \frac{\partial U}{\partial x_{i}}\right)}$

$v_{i k}=\frac{-\frac{\partial U}{\partial x_{i}}}{-\sum_{i=1}^{n}\left(x_{i k} \frac{\partial U}{\partial x_{i}}\right)}$

\section{Avaliação Cruzada (Cross Evaluation)}

A metodologia DEA concede plena liberdade às DMUs, permitindo-lhes a utilização dos pesos que melhor lhe convierem. Assim, são atribuídos pesos maiores às variáveis que possuem (em relação às outras DMUs) níveis maiores de outputs, menores de inputs; e pesos menores ou nulos às variáveis desfavoráveis à DMU em estudo. Tal liberdade diminui o poder de discriminação do método, permitindo que DMUs atinjam a eficiência devido, por exemplo, à atribuição de um valor elevado a determinado input e output, enquanto às demais varáveis é atribuído peso zero. Este fato pode ser um indicativo de que a DMU conseguiu se sobressair devido a apenas um dos aspectos analisados, não sendo de fato detentora das melhores práticas na busca pela eficiência. Esse é um grande problema no uso de DEA como ferramenta multicritério em problemas de ordenação (Soares de Mello et al., 2002), já que nesse caso seriam desconsiderados os critérios definidos pelos decisores.

Allen et al. (1997) realizaram um amplo estudo sobre diferentes metodologias para restringir os pesos em DEA. A Avaliação Cruzada (Cross Evaluation) é um desses métodos, que permite a solução do problema sem a arbitrariedade existente em outras metodologias e sem a necessidade de que se conheça previamente a importância relativa de cada variável. A metodologia, desenvolvida por Sexton et al. (1986), consiste na utilização de DEA para avaliação do conjunto, ao invés da autoavaliação realizada pelos modelos DEA clássicos. A proposta é que cada DMU seja avaliada segundo o esquema de pesos ótimos das demais DMUs, calculando-se a eficiência cruzada a partir da média de todas essas eficiências, ou seja, a avaliação de uma DMU é realizada a partir da média de suas eficiências, calculadas sob a ótica das demais DMUs (Angulo-Meza, 1998).

0 cálculo da Avaliação Cruzada clássico é iniciado com a determinação das eficiências das DMUs, o que requer a determinação de seus pesos, inclusive o das extremo-eficientes, o que é um problema, já que elas possuem múltiplas soluções. Para resolver esse problema, a abordagem clássica consiste em usar, em sequência, modelos que contenham funções objetivo secundárias para a determinação das eficiências cruzadas, posteriormente usadas na construção de uma matriz de eficiências. 0 uso de tais funções permite a obtenção de um esquema de pesos ótimo no modelo inicial, que tenha como objetivo secundário a minimização das eficiências cruzadas das outras DMUs. Além dessa formulação, dita agressiva devido à minimização da função objetivo (Sexton et al., 1986), há a formulação benevolente, cujo esquema de pesos maximiza as eficiências cruzadas das demais DMUs. Em AnguloMeza (1998), pode-se encontrar uma ampla discussão acerca do assunto.

A suavização, objeto deste estudo, é uma alternativa para a aplicação desta metodologia, pois possibilita a determinação de pesos únicos para as DMUs extremo-eficientes, obtidos pelas equações do item 5, que podem ser usados diretamente no cálculo da avaliação cruzada. No entanto, os modelos de suavização existentes até agora, que tivessem praticidade de uso, eram apenas para o modelo BCC. Ora, o modelo BCC apresenta eficiências negativas implícitas, que aparecem em situações como projeções não radiais (Gomes Júnior et al., 2013) e avaliação cruzada (Soares de Mello et al., 2002, 2013), o que faz com que apenas em raras ocasiões possa ser usado em modelos de avaliação cruzada. Assim, o desenvolvimento de um modelo de suavização para DEA-CCR passa a permitir o uso de pesos calculados por este método em modelos de avaliação cruzada. 


\section{Exemplo numérico com 2 inputs e 1 output}

Apesar de o modelo desenvolvido poder ser usado para qualquer número de variáveis, optou-se pela apresentação de um exemplo com 2 inputs e 1 output, com a finalidade de permitir a sua representação geométrica. Como já mencionado, em teoria, este modelo poderia ser tratado pelos modelos anteriores de suavização CCR, na prática, a obrigatoriedade de calcular analiticamente certas integrais de funções envolvendo raízes tornaria inviável esse cálculo.

Seja o problema apresentado na Tabela 1.

Resolvendo-o com o software SIAD (AnguloMeza et al., 2005a, b), obtém-se a solução da Tabela 2.

A Figura 1 apresenta a representação gráfica da fronteira original do problema, reduzida a 2 dimensões, através da divisão de inputs e output pelo valor do output, em cada ponto.

A DMU F está no mesmo segmento horizontal que a DMU C, sendo, portanto, fracamente eficiente. Em modelos de maior dimensão, pode-se, através de cálculos algébricos, identificar DMUs nessa

Tabela 1. Exemplo com 2 inputs e 1 output.

\begin{tabular}{cccc}
\hline DMU & Input $\mathbf{1}$ & Input 2 & Output \\
\hline A & 4 & 3 & 1 \\
B & 26 & 12 & 4 \\
C & 16 & 2 & 2 \\
D & 4 & 2 & 1 \\
E & 6 & 12 & 3 \\
F & 20 & 2 & 2 \\
\hline
\end{tabular}

Tabela 2. Eficiências do exemplo da Tabela 1.

\begin{tabular}{cc}
\hline DMU & CCR \\
\hline A & 0,857143 \\
B & 0,648649 \\
C & 1 \\
D & 1 \\
E & 1 \\
F & 1 \\
\hline
\end{tabular}



Figura 1. Representação no plano do exemplo com 2 inputs e 1 output. condição, sem grande dificuldade. 0 modelo em análise apresenta, então, 3 DMUs extremo-eficientes: C, D e E.

0 passo seguinte para a obtenção do polinômio homogêneo que representará a fronteira suavizada é a determinação do grau desse polinômio homogêneo. Para tanto, deve-se considerar ser possível eliminar o cálculo de um coeficiente, já que, ao dividir toda a equação pelo seu valor, a igualdade permanece. Convenciona-se, sem perda de generalidade, que $Z_{1}$ terá coeficiente unitário. Destaque-se ainda que o número total de coeficientes ou variáveis de decisão será a soma do $n^{\circ}$ de coeficientes do polinômio homogêneo dos outputs com o $n^{\circ}$ de coeficientes do polinômio homogêneo dos inputs.

Para se garantir que o polinômio único obtido seja homogêneo de grau 1, o grau do polinômio dos inputs deverá ser igual ao grau do polinômio dos outputs. Além disso, para que não haja inviabilidades, o grau do polinômio homogêneo a ser utilizado deverá ser superior ao número de DMUs extremoeficientes do problema investigado, acrescido de uma unidade. Destaque-se que cada DMU extremoeficiente corresponde a uma restrição de igualdade do modelo, que impõe que ela se encontre na fronteira suavizada. 0 número de variáveis de decisão deve ser maior que o número de restrições de igualdade e, como essas variáveis são os coeficientes do polinômio homogêneo aproximante, o grau deste dependerá do número de variáveis de decisão. Em resumo:

$\mathrm{N}^{\circ}$ de variáveis de decisão $>\mathrm{N}^{\circ}$ de igualdades do Modelo ( $\mathrm{N}^{\circ}$ DMUs extremo-eficientes) + 1

0 número total de coeficientes, ou variáveis de decisão, será a soma do número de coeficientes do polinômio homogêneo de inputs $\left(n_{\mathrm{p}}\right)$ com o número de coeficientes do polinômio homogêneo de outputs $\left(n_{0}\right)$. Assim, para que não haja inviabilidades:

$n_{0}+n_{1}>\mathrm{N}^{0}$ de DMUs extremo-eficientes +1

Considerando o exposto, elaborou-se um algoritmo a partir do qual foi construída a Tabela 3 , que apresenta, até o $10^{\circ}$ grau, o número de coeficientes ou termos de uma função homogênea para cada grau $g$ e número de variáveis Nvar.

No caso do exemplo, tem-se 3 DMUs extremoeficientes, $m=1$, coluna 1 e $n=2$, coluna 2 . Na Tabela 4, o grau g corresponderá à linha em que se encontre o menor valor da soma $n_{0}+n_{1}$ que satisfaça:

$n_{0}+n_{1}>3+1 \rightarrow n_{0}+n_{1}>4$

Logo, o polinômio homogêneo deverá ter grau 3 e será expresso por:

$\mathrm{U}=a x_{1}^{3}+b x_{2}^{3}+c x_{1}^{2} x_{2}+d x_{1} x_{2}^{2}-z_{1}^{3}$ 
Tabela 3. Número de coeficientes ou termos do polinômio homogêneo, para cada grau $g$ e número de variáveis nvar.

\begin{tabular}{|c|c|c|c|c|c|c|c|c|c|c|c|}
\hline & & \multicolumn{10}{|c|}{ Nvar (número de variáveis) } \\
\hline & & 1 & 2 & 3 & 4 & 5 & 6 & 7 & 8 & 9 & 10 \\
\hline & 1 & 1 & 2 & 3 & 4 & 5 & 6 & 7 & 8 & 9 & 10 \\
\hline & 2 & 1 & 3 & 6 & 10 & 15 & 21 & 28 & 36 & 45 & 55 \\
\hline o & 3 & 1 & 4 & 10 & 20 & 35 & 56 & 84 & 120 & 165 & 220 \\
\hline हًં & 4 & 1 & 5 & 15 & 35 & 70 & 126 & 210 & 330 & 495 & 715 \\
\hline$\overline{\bar{\partial}}$ & 5 & 1 & 6 & 21 & 56 & 126 & 252 & 462 & 792 & 1287 & 2002 \\
\hline$\because$ & 6 & 1 & 7 & 28 & 84 & 210 & 462 & 924 & 1716 & 3003 & 5005 \\
\hline 跑 & 7 & 1 & 8 & 36 & 120 & 330 & 792 & 1716 & 3432 & 6435 & 11440 \\
\hline$\infty$ & 8 & 1 & 9 & 45 & 165 & 495 & 1287 & 3003 & 6435 & 12870 & 24310 \\
\hline & 9 & 1 & 10 & 55 & 220 & 715 & 2002 & 5005 & 11440 & 24310 & 48620 \\
\hline & 10 & 1 & 11 & 66 & 286 & 1001 & 3003 & 8008 & 19448 & 43758 & 92378 \\
\hline
\end{tabular}

Para os valores de $m=n_{0}$ (número de outputs) e $n=n_{1}$ (número de inputs), conhecidos, procuram-se na tabela as colunas correspondentes.

Tabela 4. Escolha do grau do polinômio.

\begin{tabular}{|c|c|c|c|c|}
\hline \multirow{11}{*}{ 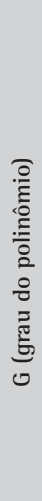 } & & $\mathrm{n}_{0}$ & $\mathrm{n}_{1}$ & $n_{0}+n_{1}$ \\
\hline & 1 & 1 & 2 & 3 \\
\hline & 2 & 1 & 3 & 4 \\
\hline & 3 & 1 & 4 & 5 \\
\hline & 4 & 1 & 5 & 6 \\
\hline & 5 & 1 & 6 & 7 \\
\hline & 6 & 1 & 7 & 8 \\
\hline & 7 & 1 & 8 & 9 \\
\hline & 8 & 1 & 9 & 10 \\
\hline & 9 & 1 & 10 & 11 \\
\hline & 10 & 1 & 11 & 12 \\
\hline
\end{tabular}

Para o problema apresentado, o modelo ficará:

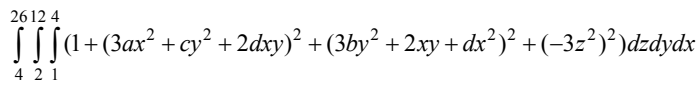

$\mathrm{S}$ a



Onde as restrições (22.a) a (22.c) são relativas às DMUs eficientes; as (22.d) e (22.e) são relativas à monotonicidade dos inputs em seus pontos de máximo. As restrições (22.f) a (22.g) garantem a monotonicidade nas DMUs extremo-eficientes. Note-se, que as restrições relativas aos outputs não foram incluídas por serem desnecessárias. Resolvendo-se a integral tripla, chega-se ao Problema de Programação Não Linear (23):

$$
\begin{aligned}
& \operatorname{Min}\left\{641539008 a^{2}+\frac{334328896}{3} d^{2}+95911200 d+\right. \\
& 287733600 a d+60241280 d b+\frac{121699864}{3}+ \\
& 60241280 a c+20512800 d c+61538400 b+ \\
& \left.3284160 c^{2}+29557440 b^{2}\right\}
\end{aligned}
$$

$\mathrm{S}$ a

$$
\begin{aligned}
& 4096 a+8 b+512 c+64 d-8=0 \\
& 64 a+8 b+32 c+16 d-1=0 \\
& 216 a+1728 b+432 c+864 d-27=0 \\
& 8000 a+8 b+800 c+80 d-8=0 \\
& 2028 a+624 c+288 d \geq 0 \\
& 432 b+676 c+624 d \geq 0 \\
& 768 a+64 c+4 d \geq 0 \\
& 48 a+16 c+4 d \geq 0 \\
& 108 a+144 c+144 d \geq 0 \\
& 12 b+256 c+64 d \geq 0 \\
& 12 b+16 c+16 d \geq 0 \\
& 432 b+36 c+144 d \geq 0
\end{aligned}
$$

Resolve-se, então, o modelo com algum software de programação matemática. Nesse caso, foi utilizado o solver do MS-Excel, obtendo-se a solução apresentada na Tabela 5.

Assim, a equação (24) representa a equação da fronteira suavizada a ser usada para substituir a fronteira original:

$\mathrm{U}=-0,00209 x_{1}^{3}+0,005618 x_{2}^{3}+$ $0,031679 x_{1}^{2} x_{2}+0,004697 x_{1} x_{2}^{2}-z_{1}^{3}$ 
Tabela 5. Valor das variáveis de decisão para o problema proposto.

\begin{tabular}{rrrr}
\hline$A$ & $b$ & $c$ & $D$ \\
\hline$-0,00209$ & 0,005618 & 0,031679 & 0,004697 \\
\hline
\end{tabular}

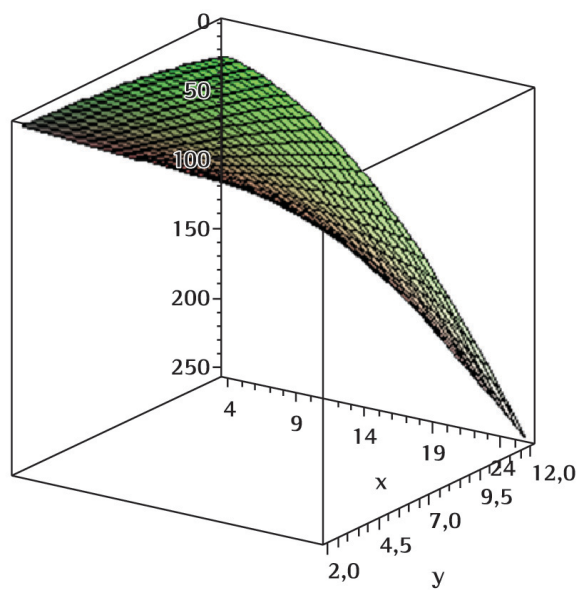

Figura 2. Gráfico da Fronteira Suavizada.

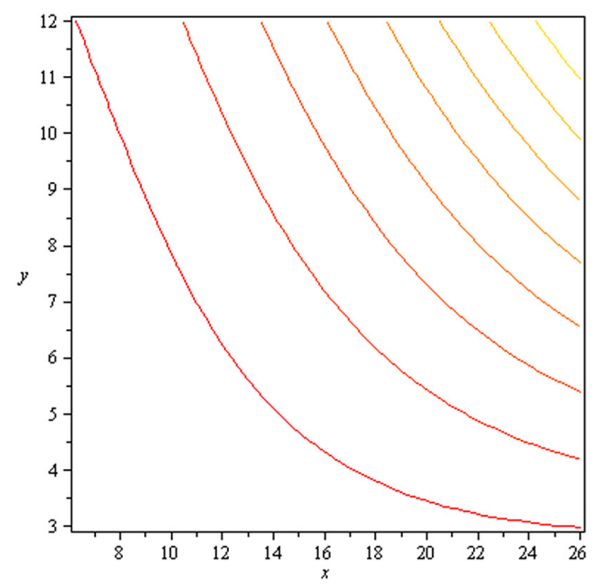

Figura 3. Curvas de Nível da Fronteira Calculada.

A Figura 2 apresenta o gráfico em 3D da fronteira calculada, construído com o software Maple. A análise da Figura 2 permite perceber que ele apresenta propriedades esperadas, já que passa pela origem e o output é uma função crescente dos inputs. Além disso, seu contorno é suave, não apresentando mudanças de concavidade.

Por sua vez, a Figura 3 mostra a curva de nível para o polinômio calculado, dentro da região de calibração definida pelos limites de $x_{1}[4,26]$ e $x_{2}$ $[2,12]$. A análise da figura permite perceber uma curva sem oscilações, com o comportamento suave esperado.
Tabela 6. Peso do Modelo Suavizado para as DMUs Extremo-eficientes.

\begin{tabular}{cccc}
\hline DMU & $\mathrm{u}_{1 \mathrm{k}}$ & $\mathrm{v}_{1 \mathrm{k}}$ & $\mathrm{v}_{2 \mathrm{k}}$ \\
\hline $\mathrm{C}$ & 0,5000 & 0,0183 & 0,3532 \\
$\mathrm{D}$ & 1,0000 & 0,1418 & 0,2165 \\
$\mathrm{E}$ & 0,3333 & 0,0619 & 0,0524 \\
\hline
\end{tabular}

Tabela 7. Eficiência das DMUs quando avaliadas pelas DMUs extremo-eficientes.

\begin{tabular}{cccc}
\hline \multicolumn{4}{c}{ Avaliada por: } \\
\hline DMU & C & D & E \\
\hline A & 0,4412 & 0,8220 & 0,8237 \\
B & 0,4241 & 0,6366 & 0,5959 \\
C & 1,0000 & 0,7404 & 0,6089 \\
D & 0,6411 & 1,0000 & 0,9461 \\
E & 0,3449 & 0,8700 & 1,0000 \\
F & 0,9316 & 0,6120 & 0,4966 \\
\hline
\end{tabular}

\subsection{Cálculo dos pesos e Avaliação Cruzada}

No exemplo desenvolvido neste estudo, obteve-se a equação (24) para a fronteira suavizada. Utilizando-se o Teorema da Função Implícita, pode-se, a partir de (16) e (17), obter os pesos das DMUs extremo-eficientes, apresentados na Tabela 6.

A Tabela 7 apresenta a eficiência de cada DMU, quando avaliada pelas DMUs extremo- eficientes.

\section{Conclusões}

Este estudo apresentou uma proposta para resolver o problema da não unicidade de pesos nas DMUs extremo-eficientes em modelos DEA CCR. A metodologia proposta foi baseada no trabalho desenvolvido por Soares de Mello (2002) e Nacif et al. (2009), que substitui a fronteira de eficiência original, que é linear por partes, por uma fronteira suavizada, com derivadas contínuas. Destaque-se que o modelo de suavização permite eliminar dois grandes problemas em DEA: regiões Pareto-ineficientes e faces de dimensão não completa.

A propriedade de proporcionalidade entre inputs e outputs dos modelos DEA CCR implica o uso de funções homogêneas de $1^{\circ}$ grau como função aproximante. A metodologia apresentada utiliza como aproximantes polinômios homogêneos que relacionem inputs e outputs.

Em modelos CCR, a complexidade para garantir a não ocorrência de grandes variações na função aproximante a ser definida requer a inclusão de restrições adicionais, relativas à monotonicidade para inputs e outputs nas DMUs extremo-eficientes. Como o interesse do estudo é calcular os trade-offs 
nas DMUs extremo-eficientes, o modelo proposto garante tal possibilidade.

0 modelo em estudo foi desenvolvido para quaisquer números de inputse outputs e interpretado geometricamente para problemas com um output (ou um input). Sem perda de generalidade, a fim de facilitar a compreensão da proposta, o problema foi apresentado a partir de um exemplo genérico de grau $n$, com 2 inputs e 1 output.

A metodologia apresentada veio preencher uma lacuna levantada em Soares de Mello et al. (2004) e Nacif et al. (2009). Além disso, conforme mostrado, a partir do modelo de suavização é possível realizar diferentes aplicações em DEA, como o cálculo da avaliação cruzada realizada.

A grande quantidade de cálculos envolvidos no modelo de suavização proposto, realizada em diferentes softwares, justifica o desenvolvimento futuro de um software para a solução de modelos de suavização, que propicie a investigação de problemas mais complexos. Destaca-se que os cálculos em si não são problemáticos, o que causa mais trabalho é a necessidade de usar ferramentas computacionais diferentes em cada etapa de resolução do modelo.

Finalmente, a suavização da fronteira DEA permite resolver alguns problemas como a multiplicidade de soluções ótimas. Por outro lado, deve-se ressaltar que há modificação, embora pequena, do conjunto de possibilidades de produção e que em determinadas situações a suavização de uma fronteira eficiente provoca perda de informação (Gorissen \& den Hertog, 2012).

\section{Referências}

Allen, R., Athanassopoulos, A., Dyson, R. G., \& Thanassoulis, E. (1997). Weights restrictions and value judgements in data envelopment analysis: evolution, development and future directions. Annals of Operations Research, 73, 1334. http://dx.doi.org/10.1023/A:1018968909638.

Andersen, P., \& Petersen, N. C. (1993). A procedure for ranking efficient units in data envelopment analysis. Management Science, 39(10), 1261-1264. http://dx.doi. org/10.1287/mnsc.39.10.1261.

Angulo-Meza, L. (1998). Data Envelopment Analysis (DEA) na Determinação da Eficiência dos Programas de PósGraduação do COPPE/UFRJ. Dissertação (Mestrado em Engenharia de Produção) - Universidade Federal do Rio de Janeiro, Rio de Janeiro.

Angulo-Meza, L., Biondi, L. N., Ribeiro, P. G., Soares de Mello, J. C. C. B., \& Gomes, E. G. (2005a). ISYDS: Integrated System for Decision Support (SIAD ${ }^{\circledR}$ : Sistema Integrado de Apoio à Decisão): a software package for data envelopment analysis Model. Pesquisa Operacional, 25(3), 493-503. http://dx.doi.org/10.1590/S010174382005000300011.

Angulo-Meza, L., Biondi, L., No, Soares de Mello, J. C. C. B., Gomes, E. G., \& Coelho, P. H. G. (2005b). Free software for decision analysis a software package for data envelopment model. In Proceedings of the 7th International Conference on Enterprise Information Systems - ICEIS (pp. 207-212).

Avellar, J. V. G., Milioni, A. Z., \& Rabello, T. N. (2007). Spherical frontier DEA model based on a constant sum of inputs. The Journal of the Operational Research Society, 58(9), 1246-1251. http://dx.doi.org/10.1057/palgrave. jors. 2602260 .

Avellar, J. V. G., Milioni, A. Z., Rabello, T. N., \& Simão, H. P. (2010). On the redistribution of existing inputs using the spherical frontier DEA model. Pesquisa Operacional, 30(1), 1-16. http://dx.doi.org/10.1590/S010174382010000100001.

Brandão, L. C. (2013). Aprimoramentos na suavização da fronteira DEA BCC. Dissertação (Mestrado em Engenharia de Produção) - Universidade Federal Fluminense, Niterói.

Brandão, L. C., \& Soares de Mello, J. C. C. B. (2013). Modelo de suavização da fronteira DEA BCC para estudar as DMUs eficientes por default. In Simpósio Brasileiro de Pesquisa Operacional, Natal.

Brandão, L. C., Andrade, F. V. S., \& Soares de Mello, J. C. C. B. (2013). UEFA Euro efficiency evaluation based on market expectations. In Proceedings of the 4th International Conference on Mathematics in Sport (pp. 32-37), Leuven.

Bregalda, P. A. O., \& Bornstein, C. (1981). Introdução à programação linear. Rio de Janeiro: Campus.

Charnes, A., Cooper, W. W., \& Rhodes, E. (1978). Measuring the Efficiency of Decision-Making Units. European Journal of Operational Research, 2(6), 429-444. http:// dx.doi.org/10.1016/0377-2217(78)90138-8.

Charnes, A., Cooper, W. W., Golany, B., Seiford, L., \& Stutz, J. (1985). Foundations of data envelopment analysis for Pareto-Koopmans efficient empirical production functions. Journal of Econometrics, 30(1-2), 91-107. http://dx.doi.org/10.1016/0304-4076(85)90133-2.

Coelli T, Rao DSP, Battese GE. An introduction to efficiency and productivity Analysis. Boston: Kluwer Academic Publishers; 1998.

Colen, E. C., Freitas, G. M., Avellar, J. V. G., \& Milioni, A. Z. (2009). On the allocation of new inputs and outputs with DEA. Engevista, 11, 4-7.

Cooper, W. W., Seiford, L. M., \& Tone, K. (2000). Data envelopment analysis: a comprehensive text with models, applications, references and DEA-Solver software. Kluwer Academic Publishers.

Cooper, W. W., Ruiz, J. L., \& Sirvent, 1. (2007). Choosing weights from alternative optimal solutions of dual multiplier models in DEA. European Journal of Operational Research, 180(1), 443-458. http://dx.doi. org/10.1016/j.ejor.2006.02.037.

Farrell, M. J. (1957). The measurement of productive efficiency. Journal of the Royal Statistical Society. Series A (General), 120(3), 253-281. http://dx.doi.org/10.2307/2343100.

Gomes, E. G., Soares de Mello, J. C. C. B., \& Lins, M. P. E. (2004). Redistribuição de inputs e outputs em modelos de análise de envoltória de dados com ganhos de soma zero. Pesquisa Operacional, 24(2), 269-284. http:// dx.doi.org/10.1590/S0101-74382004000200004.

Gomes Júnior, S. F., Soares de Mello, J. C. C. B., \& Angulo Meza, L. (2013). DEA nonradial efficiency based on vector properties. International Transactions in Operational 
Research, 20(3), 341-364. http://dx.doi.org/10.1111/ itor. 12000 .

Gorissen, B. L., \& Den Hertog, D. (2012). Approximating the Pareto set of multiobjective linear programs via robust optimization. Operations Research Letters, 40(5), 319324. http://dx.doi.org/10.1016/j.orl.2012.05.007.

Guedes, E. C. C., Milioni, A. Z., Avellar, J. V. G., \& Silva, R. C. (2012). Adjusted spherical frontier model: allocating input via parametric DEA. The Journal of the Operational Research Society, 63(3), 406-417. http://dx.doi. $\operatorname{org} / 10.1057 /$ jors.2011.42.

Lins, M. P. E., \& Angulo-Meza, L. (2000). Análise envoltória de dados e perspectivas de integração no ambiente de apoio à decisão. Rio de Janeiro: COPPE/UFRJ.

Lins, M. P. E., Gomes, E. G., Soares de Mello, J. C. C. B., \& Soares de Mello, A. J. R. (2003). Olympic ranking based on a zero sum gains DEA model. European Journal of Operational Research, 148(2), 312-322. http://dx.doi. org/10.1016/S0377-2217(02)00687-2.

Milioni, A. Z., \& Alves, L. B. (2013). Ten years of research on parametric data envelopment analysis. Pesquisa Operacional, 33(1), 89-104. http://dx.doi.org/10.1590/ S0101-74382013000100006.

Milioni, A. Z., Avellar, J. V. G., Gomes, E. G., \& Soares de Mello JCCB. An ellipsoidal frontier model: allocating input via parametric DEA. Eur J Oper Res. 2011a; 209(2):113-21. http://dx.doi.org/10.1016/j.ejor.2010.08.008.

Milioni, A. Z., Avellar, J. V. G., Rabello, T. N., \&t Freitas, G. M. Hyperbolic frontier model: a parametric DEA approach for the distribution of a total fixed input. $J$ Oper Res Soc. 2011b; 62(6):1029-37. http://dx.doi.org/10.1057/ jors.2010.24.

Nacif, F. B., Soares de Mello, J. C. C. B., \& Angulo-Meza, L. (2009). Choosing weights in optimal solutions for DEA-BCC models by means of a n-dimensional smooth frontier. Pesquisa Operacional, 29(3), 623-642. http:// dx.doi.org/10.1590/S0101-74382009000300010.

Rosen, D., Schaffnit, C., \& Paradi, J. C. (1998). Marginal rates and two dimensional level curves in DEA. Journal of Productivity Analysis, 9(3), 205-232. http://dx.doi. org/10.1023/A:1018382904489.

Sexton, T. R., Silkman, R. H., \& Hogan, A. (1986). Data envelopment analysis: critique and extensions. In R. H. Silkman. Measuring efficiency: an assessment of data envelopment analysis. New Directions for Program Evaluation, 1986(32), 73-105.

Silva, R. C., \& Milioni, A. Z. (2012). The adjusted spherical frontier model with weight restrictions. European Journal of Operational Research, 220(3), 729-735. http://dx.doi. org/10.1016/j.ejor.2012.01.064.

Silveira, J. Q., Soares de Mello, J. C. C. B., \& Angulo Meza, L. (2011). Fronteira parabólica baseada na soma constante de inputs: um estudo de ecoeficiência. In Anais do Simpósio Brasileiro de Pesquisa Operacional, Ubatuba.

Soares de Mello, J. C. C. B. (1987). Métodos variacionais em condução de calor, Dissertação (Mestrado em Matemática) - Universidade Federal Fluminense, Niterói.

Soares de Mello, J. C. C. B. (2002). Suavização da fronteira DEA com o uso de métodos variacionais, Tese (Doutorado em Engenharia de Produção) - Universidade Federal do Rio de Janeiro, Rio de Janeiro.

Soares de Mello, J. C. C. B., Gomes, E. G., Angulo Meza, L., Soares de Mello, M. H. C., \& Soares de Mello, A. J. R. (2006). Engineering post-graduate programmes: A quality and productivity analysis. Studies in Educational Evaluation, 32, 136-152. http://dx.doi.org/10.1016/j. stueduc.2006.04.005.

Soares de Mello, J. C. C. B., Gomes, E. G., Biondi, L., No, \& Lins, M. P. E. (2004). Suavização da Fronteira DEA: o caso BCC tridimensional. Investigação Operacional, 24, 89-107.

Soares de Mello, J. C. C. B., Lins, M. P. E., \& Gomes, E. G. (2002). Construction of a smoothed DEA frontier. Pesquisa Operacional, 22(2), 183-201. http://dx.doi. org/10.1590/S0101-74382002000200006.

Soares de Mello, Angulo Meza L, Silveira JQ, Gomes EG. (2013). About negative efficiencies in cross evaluation BCC input oriented models. Eur J Oper Res. 229(3):7327. http://dx.doi.org/10.1016/j.ejor.2013.02.020.

\title{
Smoothed frontier to determine a single set of weights in CCR models
}

\begin{abstract}
Over time, the number and range of applications in the DEA have increased greatly. The resulting complexity of the relationships between the multiple inputs and outputs of decision making units (DMUs) in a study hinders the use of other methodologies. This makes DEA quite attractive for identifying the performance of these DMU's. The aim of this study is to define a method of solving the ambiguity in determining the weights of extremely efficient DMUs so that each DMU has a single set of weights, allowing for determination of the relative importance of each input or output. The proposal is to replace the original CCR DEA frontier, which is piece-wise linear, with a smoothed one, which is as close as possible to the first but continuously differentiable at all points. Smoothing allows for the calculation of trade-offs in the extreme-efficient DMUs and in Cross-Evaluation, among other applications.
\end{abstract}

\section{Keywords}

DEA. Smoothed frontier. Cross-evaluation. 